Localizador: 18019 doi: $10.35366 / 93983$

\title{
Pigmentación lentiginosa mastocítica
}

\author{
Mastocytic lentiginous pigmentation \\ Eduardo Marín Hernández,* Pamela Orozco Olguín, ${ }^{\ddagger}$ \\ Ángeles Monserrat Rodríguez Sánchez, ${ }^{\S}$ Alfredo Valero Gómez ${ }^{\|}$
}

\begin{abstract}
Palabras clave:
Mastocitosis, manchas hiperpigmentadas, lesiones lentiginosas.

\section{Key words: \\ Mastocytosis, \\ hyperpigmented spots, lentiginous lesions.}

\section{* Dermatólogo y \\ dermatopatólogo Adscrito al Servicio de Dermatología. Hospital de Pediatría del Centro Médico Nacional Siglo XXI. \\ * Residente de Dermatología del Centro Dermatológico Pascua. \\ ${ }^{\S}$ Residente de Pediatría del Centro Médico Nacional Siglo XXI. \\ " Médico adscrito al Servicio de Patología. Hospital de Pediatría del Centro Médico Nacional Siglo XXI.}

Conflicto de intereses:

Recibido:

14/Marzo/2018.

Aceptado:

22/Octubre/2018.

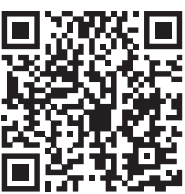

Resumen

Las mastocitosis son un grupo de enfermedades en las que existe proliferación de células mastocitarias y acumulación de ellas en la piel y tejidos extracutáneos. Paciente femenino de 10 años de edad, con lesiones hiperpigmentadas en la cara de dos años de evolución, sin mejoría. Se realizó una nueva biopsia en la que se observaron mastocitos de predominio perivascular y perianexial en escasa cantidad, por lo que se complementó realizándose CD117, que comprobó la presencia de mastocitos. Consideramos que la dermatosis de este caso tiene una presentación atípica clínica e histológica que no permite que se encuadre en las variantes clásicas de mastocitosis; por esto, proponemos el término «pigmentación lentiginosa mastocítica».

\section{ABSTRACT}

Mastocytosis is a group of diseases in which there is proliferation of mastocyte cells and accumulation of them in the skin and extracutaneous tissues. Ten-year-old female patient with hyperpigmented lesions on the face of two years of evolution, without improvement. The biopsy showed mastocytes of perivascular and periadnexal predominance in a small amount, so it was complemented with CD117, that verified the presence of mast cells. We consider that the dermatosis of this case has an atypical clinical and histological presentation that does not allow it to be framed in the classic variants of mastocytosis; for this reason, we propose the term «mastocytic lentiginous pigmentation».

\section{INTRODUCCIÓN}

$\mathrm{L}$ a mastocitosis se define como un grupo heterogéneo de síndromes clínicos caracterizados por infiltración anormal de mastocitos en varios tejidos y una liberación concomitante de mediadores químicos por estas células. En 1869, Nettleship describió la urticaria pigmentosa (UP), y en 1887, Unna reportó un aumento en el número de mastocitos en la UP. Fue en 1949 cuando Ellis describió una enfermedad sistémica asociada con hiperplasia de mastocitos. ${ }^{1}$

Se clasifica, de acuerdo con la edad de presentación, en la variante infantil y la mastocitosis de la edad adulta. En la variedad infantil, cerca de $80 \%$ de los casos se manifiestan antes del primer año de vida. Clínicamente, la mastocitosis cutánea se clasifica en urticaria pigmentosa, mastocitoma solitario, mastocitosis cutánea difusa y telangiectasia macularis eruptiva perstans. ${ }^{2}$ El diagnóstico de las mastocitosis es clínico, donde la apariencia de las lesiones cutáneas sirve para identificar el tipo, con especial importancia en el signo de Darier, que es patognomónico y positivo en más de 90\% de los casos; lo anterior se confirma con la toma de biopsia de piel, en la cual se identifica un aumento en la cantidad de células mastocíticas a nivel de la dermis superficial, sobre todo alrededor de los vasos sanguíneos y anexos, que se evidencian con tinciones especiales como el azul de toluidina o Giemsa. El manejo es sintomático y conservador, ya que la historia natural de la dermatosis es hacia la autoinvolución; asimismo, es imprescindible alertar a los padres de los detonadores que podrían agravar las lesiones cutáneas.

\section{PRESENTACIÓN DEL CASO}

Femenino de 10 años, previamente sano, sin antecedentes de importancia; inició dos años antes con manchas hiperpigmentadas en la cara, por lo que acudió con un médico particular; se le diagnosticó mastocitosis y fue tratada con ketotifeno, sin mejoría y con persistencia e incremento de las lesiones. Más tarde, se realizó una biopsia con un dermatólogo privado, la cual se reportó sin alteraciones; se concluyó el diagnóstico de 
pigmentación macular eruptiva idiopática (PMEI), por lo que se envió a esta unidad.

En la exploración física, se encontró una dermatosis diseminada a la cabeza y el tronco; la primera afectaba la cara, con predominio de las mejillas, siendo bilateral y simétrica, constituida por máculas hiperpigmentadas de color café claro, no confluentes, de bordes bien definidos, pequeñas, redondas, de aspecto lenticular; a nivel del tronco, incluía las axilas, con lesiones de las mismas características (Figura 1).

Se decidió la toma de una nueva biopsia, en la que se observó ortoqueratosis que cubría una epidermis con acantosis moderada irregular. En la dermis se identificaron con la tinción de Giemsa mastocitos de predominio perivascular y perianexial; se complementó el estudio realizándose CD117, que comprobó la presencia de mastocitos (Figura 2). Con base en estos datos clínicos e histopatológicos, concluimos que el caso se trataba de una mastocitosis; sin embargo, este cuadro no se logra englobar en las características descritas usuales de este grupo de patologías, teniendo una imbricación de la expresión clínica, la cual es atípica, y la histología que se aprecia en la telangiectasia eruptiva perstans, por lo que optamos por un nombre descriptivo de la misma: pigmentación lentiginosa mastocítica. Se inició el manejo con ketotifeno (1 mg por las noches), ciclo corto con esteroide de baja potencia del tipo hidrocortisona al 1\% en lesiones de mayor dimensión, de lunes a viernes por seis semanas; hubo disminución del tono hiperpigmentado. Se envió a Hematología, que descartó involucramiento sistémico.
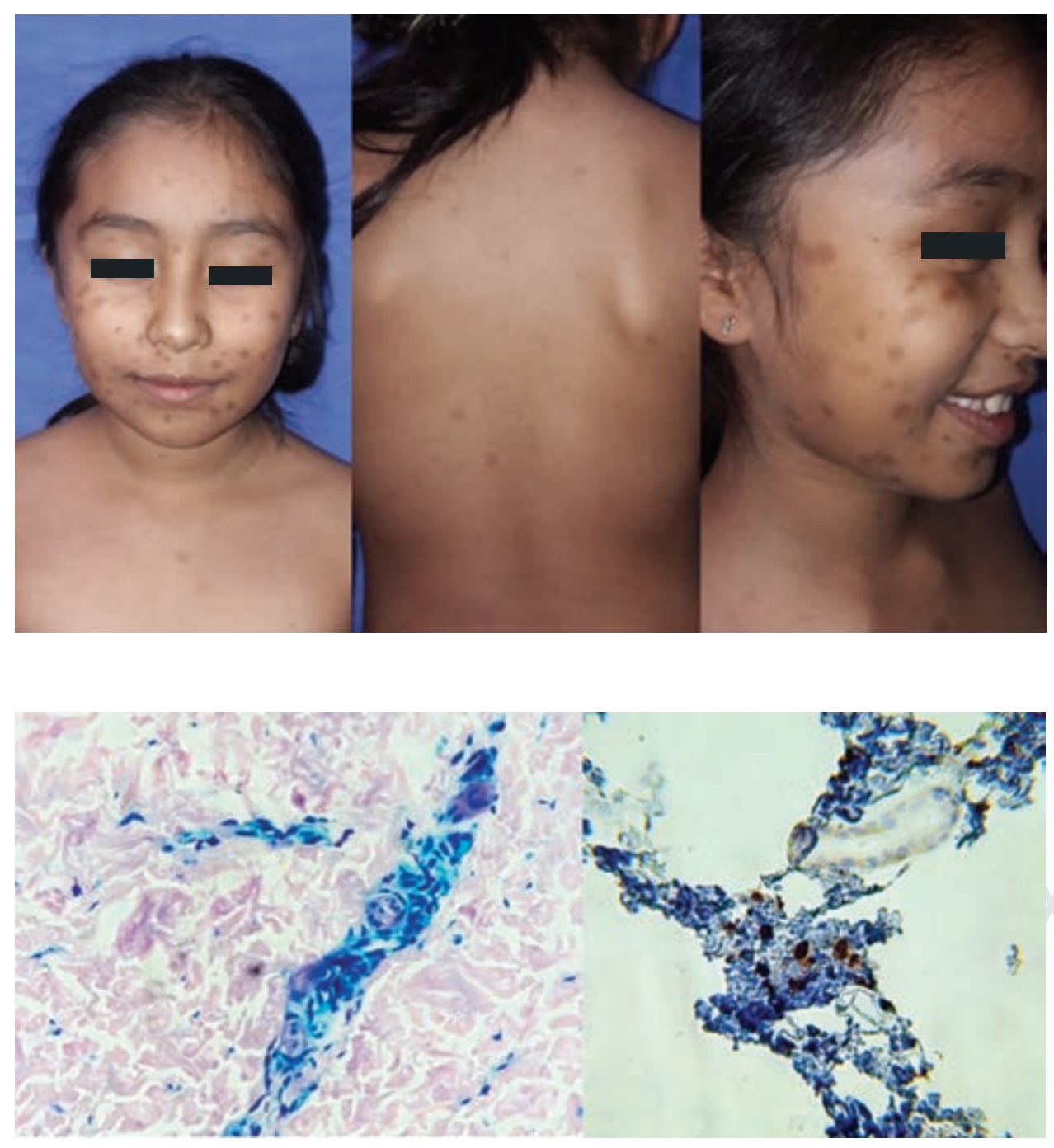

Figura 1.

Máculas lenticulares cafés con predominio facial.

Figura 2.

40x tinción de Giemsa con evidencia de mastocitos, de predominio perivascular y CD117 positivo para células mastocíticas con distribución perivascular. 


\section{DISCUSIÓN}

Las mastocitosis son un grupo de enfermedades en las que existe proliferación de células mastocitarias y acumulación de ellas en la piel y tejidos extracutáneos. Los mastocitos se originan en la médula ósea a partir de un precursor hematopoyético pluripotencial y circulan en la sangre como células CD34+; posteriormente, atraviesan el endotelio para alojarse en los tejidos periféricos como la piel, el pulmón y el intestino, donde se diferencian y maduran. Allí forman gránulos de secreción que expresan en su superficie receptores para IgE. ${ }^{1}$

Hasta el momento se desconoce la causa del aumento del número de mastocitos, pero se han propuesto diferentes hipótesis: mutación del protooncogén c-kit; expresión del factor de crecimiento de los mastocitos y del c-kit; apoptosis, alteraciones cromosómicas y fenotipo de los mastocitos. ${ }^{2}$

Según la clasificación de la OMS de 2001, las mastocitosis se dividen en procesos limitados a la piel, denominados «mastocitosis cutáneas» y aquéllas que comprometen otros tejidos como médula ósea, huesos, hígado, bazo y ganglios linfáticos. ${ }^{3}$

La mastocitosis cutánea, a su vez, se clasifica en urticaria pigmentosa (UP), mastocitoma solitario (MS), mastocitosis cutánea difusa (MCD) y telangiectasia macularis eruptiva perstans (TMEP). ${ }^{4}$

Urticaria pigmentosa. Es la forma clínica más frecuente; representa $70-90 \%$ de los casos y afecta sobre todo a lactantes y niños. Las lesiones pueden aparecer al nacimiento, pero lo más habitual es la ocurrencia de diferentes brotes durante los primeros meses hasta aproximadamente los dos años. Las lesiones se componen de máculas, pápulas, placas o nódulos mal delimitados, de tamaño variable y de color marrón o violáceo. En la exploración física, cuando se frotan las lesiones se produce eritema, habón o ampolla, lo que constituye el signo de Darier. Es raro que se afecten las palmas, plantas o mucosas; en general, las lesiones se localizan en el tronco, con una distribución simétrica. Cuando las lesiones se encuentran muy diseminadas y en abundante cantidad, se le ha denominado «baby leopard». ${ }^{5}$ El síntoma más común es el prurito. En niños, las lesiones autoinvolucionan y desaparecen al llegar a la adolescencia en el 50\% de los casos, y el $25 \%$ al llegar a la vida adulta. ${ }^{6,7}$

Mastocitoma solitario. Representa $10-35 \%$ de las mastocitosis cutáneas. Es más común que se presente en los primeros meses de vida. Las lesiones se componen de nódulos o placas infiltradas únicas con superficie de coloración parduzca o amarillenta, principalmente en muñecas, codos y tronco, con signo de Darier positivo, redonda u ovalada, con un diámetro variable de entre uno y cinco centímetros. El pronóstico es bueno. ${ }^{8}$

Telangiectasia macular eruptiva persistente. Se caracteriza por la aparición de máculas hiperpigmentadas telangiectásicas que, a diferencia de otras mastocitosis, presentan respuesta urticariforme escasa. Se localizan con frecuencia en la parte alta de tronco. Tiene evolución benigna y suele ocurrir sobre todo en adolescentes y adultos. ${ }^{9}$

Mastocitosis cutánea difusa. Representa el 1-3\% de los casos de mastocitosis cutánea. Se considera la forma clínica más rara y se caracteriza por la presencia de infiltración difusa de la piel en todo su espesor. Los pacientes pueden estar asintomáticos al nacimiento, desarrollando en los primeros meses un engrosamiento de la piel, que adquiere una textura similar a la piel de naranja. Las lesiones se acentúan en las áreas de flexión, con una coloración normal o amarillenta. Puede haber desarrollo de ampollas grandes y tensas sobre la superficie de la piel afectada. En esta forma clínica es común la presencia de síntomas sistémicos como rubor intenso, broncoespasmo y diarrea. Cursa con prurito intenso por la infiltración tan importante $y$, en particular, es sensible a PUVA. ${ }^{8,9}$

El diagnóstico se realiza con la presunción clínica y la semiología, y se confirma histopatológicamente. El estudio histopatológico con hematoxilina y eosina muestra aumento del infiltrado mastocitario en las papilas dérmicas, con predominio perivascular. La tinción de azul de toluidina y el Giemsa permiten identificar la metacromasia de los gránulos mastocitarios. ${ }^{10}$

El tratamiento en general de las mastocitosis va encaminado a evitar la degranulación de los mastocitos; es decir, evitar factores detonantes como el ejercicio, el baño caliente, la fricción, o fármacos como ácido acetilsalicílico y AINE, entre otros; los esteroides tópicos se indican para mejorar las lesiones cutáneas y el número de mastocitos. ${ }^{7}$ Particularmente en nuestra paciente, se indicó ketotifeno, sin lograr modificar la clínica pigmentaria de las lesiones, aun cuando ella no presentaba signo de Darier; sin embargo, la pigmentación persistió, lo que motivó el uso de un esteroide de baja potencia, con lo que se logró una disminución del tono café de las máculas en la piel.

Existen diversos diagnósticos diferenciales con respecto a las manchas hiperpigmentadas de predominio en la cara; por ejemplo (Tabla 1): ${ }^{11}$

Hiperpigmentación postinflamatoria: es la causa más frecuente de la hiperpigmentación adquirida benigna y transitoria en la infancia; aparece tras la resolución de una lesión eritematosa. Los individuos de piel oscura son más 
Tabla 1. Diagnóstico diferencial de las máculas hiperpigmentadas en la infancia.

$\begin{array}{lllll}\text { Entidad } & \text { Clave clínica } & \text { Pruebas complementarias } & \begin{array}{c}\text { Predominio } \\ \text { facial }\end{array} & \begin{array}{c}\text { Compromiso } \\ \text { sistémico }\end{array} \\ \text { Evolución }\end{array}$

Modificado de Irvine et al. ${ }^{10}$

susceptibles. Se manifiesta como máculas hiperpigmentadas que varían de color desde marrón oscuro a gris azulado. Estas manchas pueden persistir por meses o años. ${ }^{12}$

Dermatosis purpúricas pigmentadas: son un grupo de enfermedades poco frecuentes durante la edad pediátrica, caracterizadas por una erupción petequial y máculas hiperpigmentadas; se observan los característicos «puntos en pimienta de cayena», con predominancia en las extremidades inferiores y de manera simétrica. Histológicamente, se observa el fenómeno de capilaritis en la dermis superficial, con extravasación de hematíes y hemosiderina, sin signos de necrosis fibrinoide ni vasculitis. El curso evolutivo es crónico, alternando periodos de mejoría y empeoramiento. ${ }^{13}$

La pigmentación macular eruptiva idiopática es una enfermedad poco frecuente caracterizada por máculas hiperpigmentadas de color grisáceo o pizarra, no confluentes y asintomáticas, que comprometen el tronco, el cuello y las extremidades proximales, respetando cara; ocurre en niños y adolescentes sin antecedentes de inflamación previa ni exposición a drogas. En la histopatología se observa una hiperpigmentación de las células basales de la epidermis y melanófagos en la dermis, sin signos de infiltrado inflamatorio liquenoide y recuento normal de mastocitos. La evolución de esta enfermedad es autorresolutiva en un periodo de seis meses a un año. ${ }^{14}$

Nuestra paciente presentaba las manchas hiperpigmentadas sobre todo en la cara, y escasas en el tronco, de aspecto lentiginoso, en tono café marrón, con signo de Darier ausente. Este signo puede no estar positivo en todos los casos; su presencia se ha reportado en 88 a 92\% de ellos. El hallazgo histopatológico característico es la presencia de mastocitos en la dermis superficial y media; en algunas variantes, como en TMEP y ciertos casos de urticaria pigmentosa con expresión clínica macular, se pueden observar escasas células mastocíticas y una distribución perivascular, que son más evidentes con la tinción de azul de toluidina, Giemsa, y aún más específicamente, con inmunohistoquímica con el marcador CD117. ${ }^{15} \mathrm{El}$ CD117 ha sido evaluado como marcador de mastocitosis para diferenciarlos de otras lesiones, lo que hace más fácil el diagnóstico, en especial, en casos donde hay menor número de mastocitos. ${ }^{16,17}$

\section{CONCLUSIONES}

Consideramos que el caso corresponde a una variante de mastocitosis atípica, con signo de Darier ausente e histopatología paucimastocítica de predominio perivascular, como la que encontramos en la telangiectasia macular 
eruptiva perstans; en la serie de casos planteados como problema de diagnóstico, el uso de la inmunohistoquímica corroboró el diagnóstico de mastocitosis. ${ }^{17}$ La dermatosis de este caso tiene una presentación atípica clínica e histológica que no permite que se encuadre en las variantes clásicas de mastocitosis; por esto, proponemos el término «pigmentación lentiginosa mastocítica», que describe su comportamiento clínico e histológico.
Correspondencia:

Eduardo Marín-Hernández

Hospital de Pediatría CMN Siglo XXI.

Av. Cuauhtémoc Núm. 330,

entre Dr. Marquez y Eje 3 Sur,

Col. Doctores, 06720 ,

Del. Cuauhtémoc, Ciudad de México.

Teléfono: 5627 6900, ext. 22268

E-mail: emarinh1973@yahoo.com.mx

\section{BIBLIOGRAFÍA}

1. Molina-Garrido MJ, Mora A, Guillén-Ponce C, Guirado-Risueño M, Molina MJ, Molina MA et al. Mastocitosis sistémica: Revisión sistemática. An Med Interna (Madrid). 2008; 25 (3): 134-140.

2. Maldonado-Colín G, Campos-Cabrera BL, García-Romero MT. Mastocitosis cutánea: lo que el pediatra debe saber. Alerg Asma Inmunol Pediatr. 2015; 24 (3): 78-85.

3. Pérez-Elizondo AD, Zepeda-Ortega B, Pino-Rojas GT. Urticaria pigmentosa: un enfoque actual. Rev Alerg Mex. 2009; 56 (4): 124-135.

4. Bajoghli AA, Blankenship CM. Picture of the month. Urticaria pigmentosa. Arch Pediatr Adolesc Med. 2008; 162 (4): 383-384.

5. Rueda ML, Yarza ML, Colina V, Gallego S, Pereira S, Navacchia $D$ et al. Mastocitosis cutánea: revisión de 10 años de experiencia en el Servicio de Dermatología del Hospital General de Niños Pedro de Elizalde. Dermatología Argentina. 2010; 17 (1): 32-39.

6. Guerouaz N, Hassam B. Cutaneous mastocytosis: baby leopard appearance. Pan Afr Med J. 2013; 16: 146.

7. Herrera CE, Moreno CA, Requena CL et al. Mastocitosis, dermatopatología: correlación clínico-patológica. España: Grupo Menarini; 2007. pp. 364-368.

8. Fábrega J, Moraga F. Mastocitosis. Protocolos de dermatología. 2. ${ }^{a}$ ed. Barcelona: Asociación Española de Pediatría; 2007. pp. 179-184.

9. Lange M, Niedoszytko M, Renke J, Glen J, Nedoszytko B. Clinical aspects of paediatric mastocytosis: a review of 101 cases. J Eur Acad Dermatol Venereol. 2013; 27 (1): 97-102.
10. Castells M, Metcalfe DD, Escribano L. Guidelines for the diagnosis and treatment of cutaneous mastocytosis in children. Am J Clin Dermatol. 2011; 12 (4): 259-270.

11. Clinical manifestations, pathogenesis, and classification of mastocytosis (cutaneous and systemic). [Acceso: 12 de enero de 2011]. Disponible en: www.uptodate.com/contents/ clinical-manifestations-pathogenesis-and-classification-ofmastosytosis-cutaneous-and-systemic

12. Irvine A, Hoeger P, Yan A. Disorders of hyperpigmentation. Harper's Textbook of pediatric dermatology. $3^{\text {rd }}$ ed. Editorial Wiley-Blackwell; 2011.

13. Giacaman A, Knopfel N, Campos M, Martin-Santiago A. Acquired facial hyperpigmented macules in children: 3 new cases. Actas Dermosifiliogr. 2016; 107: 81-83.

14. Coulombe J, Jean SE, Hatami A, Powell J, Marcoux D, Kokta V et al. Pigmented purpuric dermatosis: Clinico-pathologic characterization in a pediatric series. Pediatr Dermatol. 2015; 32: 358-362.

15. Jang KA, Choi JH, Sung KS, Moon KC, Koh JK. Idiopathic eruptive macular pigmentation: report of 10 cases. J Am Acad Dermatol. 2001; 44 (2 Suppl): 351-353.

16. Kiszewski AE, Durán-Mckinster C, Orozco-Covarrubias L, Gutiérrez-Castrellón P, Ruiz-Maldonado R, Kiszewski AE1 et al. J Eur Acad Dermatol Venereol. 2004; 18 (3): 285-290.

17. Pătraşcu V, Enache AO, Ciurea RN, Georgescu CC, Vîlcea AM, Stoica LE et al. Cutaneous mastocytosis, problems of clinical diagnosis of four cases. Rom J Morphol Embryol. 2014; 55 (3): 965-971. 